\title{
Association of GPx1 P198L and CAT C-262T Genetic Variations With Polycystic Ovary Syndrome in Chinese Women
}

Yuan Sun ${ }^{1,2}$, Suiyan $\mathrm{Li}^{2}$, Hongwei Liu ${ }^{3}$, Yan Gong ${ }^{3}$, Huai Bai ${ }^{1}$, Wei Huang $^{3}$, Qingqing Liu ${ }^{1}$,
Linbo Guan ${ }^{1}$ and Ping Fan ${ }^{1 *}$

${ }^{1}$ Laboratory of Genetic Disease and Perinatal Medicine, Key Laboratory of Birth Defects and Related Diseases of Women and Children, Ministry of Education, West China Second University Hospital, Sichuan University, Chengdu, China, ${ }^{2}$ School of Life Science and Engineering, Southwest Jiaotong University, Chengdu, China, ${ }^{3}$ Department of Obstetrics and Gynecology, West China Second University Hospital, Sichuan University, Chengdu, China

Background: Oxidative stress plays an important role in the pathogenesis of polycystic ovary syndrome (PCOS). Glutathione peroxidase 1 (GPX1) and catalase (CAT) are the major intracellular antioxidant enzymes that can detoxify hydrogen peroxide into water, preventing cellular injury from reactive oxygen species. The aim of the present study was to investigate the association of GPx1 P198L (Pro198Leu, C559T, rs 1050450) and CAT C-262T (rs1001179) genetic polymorphisms with the risk of PCOS and evaluate the effects of the genotypes on clinical, hormonal, metabolic and oxidative stress parameters in Chinese women.

Methods: This is a case-control study of 654 patients with PCOS and 535 controls. The GPx1 P198L, CAT C-262T, and superoxide dismutase 2 (SOD2) A16V genotypes were determined by polymerase chain reaction amplification and restriction analysis. Clinical, hormonal, metabolic and oxidative stress parameters were also analyzed.

Results: The frequencies of the $P L+L L$ genotype (14.1 vs. 8.4\%) and $L$ allele (7.3 vs. $4.4 \%$ ) of GPX1 P198L polymorphism were significantly higher in patients with PCOS than in control subjects. Genotype $(P L+L L)$ remained a significant predictor for PCOS in prognostic models including age, body mass index (BMI), insulin resistance index, total cholesterol, triglycerides, high-density lipoprotein-cholesterol, and low-density lipoprotein-cholesterol as covariates (OR $=2.105,95 \% \mathrm{Cl}$ : $1.330-3.331, P$ $=0.001$ ). Patients carrying the $L$ allele had relatively high average ovarian volume, waist circumference, and malondialdehyde levels $(P<0.07)$ compared with patients with the $P P$ genotype. We also demonstrated that the subjects with both GPx1 $L$ and SOD2 $A$ alleles further increase the risk of PCOS compared with the individuals carrying the $P P / W$ genotype after adjusting for age and $\mathrm{BMI}(\mathrm{OR}=5.774,95 \% \mathrm{Cl}: 2.243-14.863$, $P<0.001)$. However, no significant differences were observed in the frequencies of the CAT C-262T genotypes and alleles between PCOS and control groups.

Conclusions: The GPX1 P198L, but not CAT C-262T, genetic polymorphism is associated with the risk of PCOS in Chinese women.

Keywords: polycystic ovary syndrome, glutathione peroxidase 1, catalase, superoxide dismutase 2, genetic polymorphism, oxidative stress 


\section{INTRODUCTION}

Polycystic ovary syndrome (PCOS) is a heterogeneous female endocrine-reproductive-metabolic abnormality with a worldwide prevalence of $4-21 \%$ in reproductive-aged women, depending on diagnostic criteria $(1,2)$. In addition to reproductive disorders, PCOS is often associated with overweight, obesity and central obesity, dyslipidemia, insulin resistance (3-5), increased oxidative stress, chronic low-grade inflammation (6-8), elevated risks of metabolic syndrome, impaired glucose tolerance, type 2 diabetes, and future cardiovascular diseases $(3,9,10)$. The etiology of PCOS is unclear, but studies have suggested that PCOS appears to have a complex, multifactorial etiology resulting from the interactions between genetic, environmental, and intrauterine factors (3).

Glutathione peroxidase (GPx) 1, the most common isoform of the GPx family, is a selenium-dependent antioxidant enzyme that can reduce free hydrogen peroxide $\left(\mathrm{H}_{2} \mathrm{O}_{2}\right)$ to water by using glutathione (GSH) as a reducing substrate in human cells. Thus, GPx1 plays an important role in the detoxification of $\mathrm{H}_{2} \mathrm{O}_{2}$ and a wide range of organic peroxides $(11,12)$. GPX1 P198L (Pro198Leu, C559T, rs1050450) polymorphism is a $C$ to $T$ substitution in exon 2 of GPx1 gene, leading to an amino acid change from proline (Pro) to leucine (Leu) at codon 198 (12), which results in a reduced enzyme activity (13). GPxl gene P198L polymorphism has been reported to be related with some oxidative stress-related diseases, such as lung cancer (12), breast cancer (13), bladder cancer (14), diabetic peripheral neuropathy (11), and inflammatory bowel disease (15).

Catalase (CAT) is another antioxidant enzyme that detoxifies $\mathrm{H}_{2} \mathrm{O}_{2}$ into oxygen and water, preventing cellular injury from reactive oxygen species (16). Hereditary catalase deficiencies have been associated with increased risk of diabetes (17). A common functional single-nucleotide polymorphism (SNP) C$262 \mathrm{~T}$ (rs 1001179) in the promoter region of the human CAT gene influences transcription factor binding, resulting in a lower $C A T$ gene expression in the $C$ allele compared to the $T$ allele (18). However, the association between the C-262T polymorphism and CAT activity is contradictory, but most report a decrease in activity associated with the $T$ allele $(19,20)$. The $C-262 T$ polymorphism has been reported to be related to some human diseases, such as prostate cancer (21), male infertility (22), and glucose and lipid abnormalities in patients with type 2 diabetes or hyperlipidemia $(19,20)$.

Oxidative stress plays a key role in the occurrence and development of PCOS (8), so understanding the function of SNPs involved with antioxidant enzymes is fundamental. Our previous studies have demonstrated that 3 SNPs of the antioxidant enzymes, the Q192R variant in the paraoxonase 1 (PON1) gene, the G994T polymorphism in the platelet-activating factor acetylhydrolase $(P A F-A H)$ gene, and the $A 16 \mathrm{~V}$ variant in the superoxide dismutase 2 (SOD2) gene are associated with the risk of PCOS in Chinese women (23-25). Because GPx1 and CAT are important intracellular antioxidant enzymes and play an important role in maintaining the dynamic balance of oxidative stress interacting with $\operatorname{SOD} 2(26,27)$, we hypothesized that the functional SNPs, the P198L in GPx1 gene and the C-262T in CAT gene, may be associated with PCOS. Salahshoor et al. (28) reported that the GPX1 P198L and the CAT C-262T SNPs were not associated with the risk of PCOS in the Kurdish population, but it remains unknown whether there are any relationships between these 2 gene polymorphisms and PCOS in Chinese women. In the present study, we investigated the relationships between GPX1 P198L and CAT C-262T variants and the risk of PCOS, evaluated the effects of the genotypes on clinical, hormonal, metabolic and oxidative stress parameters, and analyzed the associations of the genotype combinations of GPx1 P198L and SOD2 A16V with PCOS in well-characterized Chinese women using relatively large sample sizes.

\section{SUBJECTS AND METHODS}

\section{Study Subjects}

This was a case-control study with 654 cases and 535 controls. Women with or without PCOS aged 17-40 years were recruited from 2006-2019 from the Outpatient Clinic of Reproductive Endocrinology of the West China Second University Hospital. All of the participants provided their written informed consent, and the study was approved by the Institutional Review Board of the West China Second University Hospital, Sichuan University (2014-014 to PF).

Each patient with PCOS met the revised 2003 Rotterdam ESHRE/ASRM consensus criteria (29). Oligo-ovulation or anovulation (OA) was assessed as irregular menstrual cycles (i.e., $<21$ or $>35$ days or $<8$ cycles per year) (30). Clinical or biochemical hyperandrogenism (HA) was assessed by hirsutism with a modified Ferriman-Gallwey (F-G) score of more than 6, clinical presence of obvious acne, total testosterone (TT) levels and/or free androgen index (FAI) above the 95th percentile of the levels $(\mathrm{TT} \geq 2.60 \mathrm{nmol} / \mathrm{L}$, FAI $\geq 9.5$ ) detected in a group of normal menstruating women with normal cycles $(4,23,31)$. Polycystic ovaries (PCOs) were confirmed if there were 12 or more follicles in each ovary measuring $2-9 \mathrm{~mm}$ in diameter and/or increased ovarian volume $(>10 \mathrm{~mL})$ by ultrasonic examination. The diagnosis of PCOS was based on a patient having 2 of these 3 findings, with $\mathrm{HA}$ as an essential condition for women aged $<20$ years $(30,32)$ and exclusion of other etiologies, such as androgen-secreting tumors, congenital adrenal hyperplasias, and Cushing syndrome. All the controls had regular menstrual cycles (between 21 and 35 days), exhibited normal circulating androgen levels, did not show hirsutism or obvious acne on physical examination, and had normal ovarian morphology as determined by ultrasound.

None of the subjects had clinically evident acute or chronic diseases, such as infection, tumors, cardiovascular disease, thyroid dysfunction, autoimmune diseases, endometriosis, premature ovarian insufficiency, hyperprolactinemia, or hypogonadotropic hypogonadism.

The subjects were also excluded if they had one of the following interferential factors: (i) taking hormones or medication (such as oral contraceptives, metformin, etc) known to affect the metabolism of carbohydrates or lipids within 3 months before the study; (ii) being pregnant or in the luteal phase 
because progesterone was reported to influence oxidative stress $(33,34)$; and (iii) smoking.

Clinical and anthropometric variables, including waist circumference, hip circumference, waist-to-hip ratio, body mass index (BMI, $\mathrm{kg} / \mathrm{m}^{2}$ ), systolic and diastolic blood pressure (SBP and DBP), and the degree of acne and hirsutism were measured or evaluated as described previously $(35,36)$ in all subjects. Ultrasound ovarian volume $(\mathrm{ml})$ on each side was calculated using the formula for the volume of an ellipsoid (37): $0.523 \times$ length $(\mathrm{cm}) \times$ width $(\mathrm{cm}) \times$ thickness $(\mathrm{cm})$.

Blood samples were obtained in the morning after overnight fasting, placed on ice immediately and centrifuged at $1,500 \mathrm{~g}$ for $15 \mathrm{~min}$ at $4^{\circ} \mathrm{C}$ within $2 \mathrm{~h}$. Plasma or serum were stored at $-80^{\circ} \mathrm{C}$, and blood cells were stored at $4^{\circ} \mathrm{C}$.

\section{Genotype Analysis}

Genomic DNA was extracted from whole blood samples as previously described $(24,25)$. The GPx1 P198L and CAT C-262T genotypes were determined by polymerase chain reaction (PCR) amplification and restriction analysis according to the method of Kasznicki et al. (38) with some modification. A total volume of $25 \mu \mathrm{l}$ containing $2.5 \mu \mathrm{l}$ of $10 \mathrm{X}$ PCR buffer, $200 \mu \mathrm{M}$ each of dNTP, $1.5 \mathrm{mM} \mathrm{MgCl}_{2}$, and $0.75 \mathrm{U}$ of Taq polymerase (Thermo Fisher Scientific Inc. Vilnius, Lithuania), $1.5 \mu \mathrm{l}$ of genomic DNA template (about 30-80 ng), $0.30 \mu \mathrm{M}$ of each primer: forward $5^{\prime}$-GTGCCCCTACGCAGGTACAG-3' and reverse $5^{\prime}$-GGACATACACACAGTTCTGCTGAC-3' for the P198L genotype (designed by Primer-BLAST); forward 5'-CTGATAACCGGGAGCCCCGCCCTGGGTTCGGATAT-

$3^{\prime}$ and reverse 5'-CTAGGCAGGCCAAGATTGGAAGCCC AATGG-3' for the $C-262 T$ genotype were used (39). The PCR was performed as follows: pre-denaturation at $95^{\circ} \mathrm{C}$ for $5 \mathrm{~min}$, followed by 33 (GPx1)/29 (CAT) cycles of $45 \mathrm{~s}$ at $95^{\circ} \mathrm{C}, 45 \mathrm{~s}$ at $66^{\circ} \mathrm{C}(\mathrm{GPx} 1) / 70^{\circ} \mathrm{C}(\mathrm{CAT})$, and $45 \mathrm{~s}$ at $72^{\circ} \mathrm{C}$, and ending with a single 7 min extension step at $72^{\circ} \mathrm{C}$. Five microliter of the $P 198 \mathrm{~L}$ (319 bp) or C-262T (190 bp) PCR products were digested with $8 \mathrm{U}$ of ApaI or $5 \mathrm{U}$ of EcoRV (New England Biolabs, Inc.) in $10 \mu \mathrm{l}$ of reaction volume for $1-3 \mathrm{~h}$ at 25 or $37^{\circ} \mathrm{C}$, respectively, and digestion resulted in 239- and 80-bp fragments for the $198 \mathrm{P}$ allele and in a non-digested 319-bp fragment for the $198 \mathrm{~L}$ allele, 157- and 33-bp fragments for the $-262 \mathrm{C}$ allele, and a non-digested 190-bp fragment for the $-262 T$ allele. The products were analyzed by electrophoresis on a 2.5 or $3.5 \%$ agarose gel and visualized by staining with Genecolour fluorescent dye. SOD2 A16V polymorphisms (C47T, rs4880) were measured as described previously (23). For the purpose of genotyping quality control, more than $30 \%$ of DNA samples were randomly genotyped again by a different operator and the results of the two genotyping were identical.

\section{Analysis of Hormonal, Metabolic, and Oxidative Stress Parameters}

Serum follicle-stimulating hormone (FSH), luteinizing hormone (LH), TT, sex hormone-binding globulin (SHBG), estradiol $\left(\mathrm{E}_{2}\right)$, total cholesterol (TC), triglyceride (TG), HDL-cholesterol (HDLC), LDL-cholesterol (LDL-C), total oxidant status (TOS), total antioxidant capacity (T-AOC) and malondialdehyde (MDA) levels, plasma insulin and glucose concentrations, as well as free androgen index (FAI), homeostatic model assessment of insulin resistance (HOMA index), and oxidative stress index (OSI) were measured or assessed as described previously $(4,6,23)$. The intraand inter-assay coefficients of variation for all measurements were $<5$ and $10 \%$, respectively.

\section{Statistical Analyses}

Data were presented as the mean \pm standard deviation (SD). Differences in variables were evaluated by the independent sample $t$-test between PCOS and control subjects. Variables with asymmetric distribution were evaluated by non-parametric tests (Mann-Whitney $U$-test). Chi-squared $\left(x^{2}\right)$ analysis was used to test deviations of genotype distribution from Hardy-Weinberg and to determine allele or genotype frequencies between patients and controls. Odds ratios (ORs) and 95\% confidence intervals (CIs) were calculated to test relative risk for PCOS associated with the gene variants by $x^{2}$ analysis and logistic regression methods. Analysis of covariance was used to estimate the differences in metabolic and oxidative stress parameters between 2 groups after correction for differences in covariates, such as age and BMI. A $P$-value $<0.05$ was considered to be statistically significant. All statistical analyses were performed using the Statistical Program for Social Sciences (SPSS) 13.0 for Windows (Chicago, IL, USA).

\section{RESULTS}

\section{Clinical and Biochemical Characteristics of the Study Population}

As shown in Table 1, BMI was significantly increased and age was significantly decreased in the PCOS group compared with the control group.

Waist circumference, waist-to-hip ratio, F-G score, acne grade score, DBP, average ovarian volume, TT levels, the ratio of $\mathrm{LH}$ to FSH, FAI, fasting insulin concentration, HOMA index, TG, TC, LDL-C, TOS, T-AOC, and MDA levels, and OSI were significantly higher, whereas SHBG and HDL-C levels were significantly lower in the PCOS group compared with the control group after adjusting for age and BMI (Table 1).

\section{Distribution of GPX1 P198L and CAT C-262T Genotypes and Alleles}

Genotypic and allelic frequencies of GPx1 P198L and CAT C$262 T$ polymorphisms are summarized in Table 2. Genotypic distributions were in Hardy-Weinberg equilibrium in patients with PCOS and the control women (all $P>0.05$ ). The frequencies of the $P L+L L$ genotype (14.1 vs. $8.4 \%$ ) and $L$ allele (7.3 vs. $4.4 \%)$ of GPx1 P198L polymorphism were significantly higher in the PCOS group compared with the control group $(\mathrm{OR}=1.671,95 \%$ CI: $1.190-2.346, P=0.003)$. Logistic regression analysis adjusted for age, BMI, HOMA-IR, TG, TC, HDL-C and LDL-C showed that the genotype $(P L+L L)$ remained a significant predictor for PCOS $(\mathrm{OR}=2.105,95 \% \mathrm{CI}: 1.330-3.331, P=0.001)$. No significant differences were found in the frequencies of CAT C$262 T$ genotypes and alleles between PCOS and control groups (Table 2, $P>0.05$ ). 
TABLE 1 | Clinical, hormonal, metabolic, and oxidative stress parameters in PCOS patients and control women.

\begin{tabular}{|c|c|c|c|c|}
\hline & $\begin{array}{l}\text { Controls } \\
(n=535)\end{array}$ & $\begin{array}{c}\text { PCOS } \\
(n=654)\end{array}$ & $P$ & $P^{a}$ \\
\hline Age (years) & $28.15 \pm 4.15$ & $25.00 \pm 4.21$ & 0.000 & \\
\hline BMI $\left(\mathrm{kg} / \mathrm{m}^{2}\right)$ & $21.12 \pm 2.86$ & $23.24 \pm 4.27$ & 0.000 & \\
\hline Waist circumference (cm) & $73.67 \pm 8.38$ & $80.00 \pm 11.42$ & 0.000 & 0.000 \\
\hline Waist-to-hip ratio & $0.81 \pm 0.06$ & $0.85 \pm 0.07$ & 0.000 & 0.000 \\
\hline F-G score & $0.25 \pm 0.73$ & $1.74 \pm 2.06$ & 0.000 & 0.000 \\
\hline Acne grade score & $0.14 \pm 0.35$ & $0.65 \pm 0.91$ & 0.000 & 0.000 \\
\hline $\mathrm{SBP}(\mathrm{mmHg})$ & $112.93 \pm 11.48$ & $114.84 \pm 10.72$ & 0.004 & 0.976 \\
\hline $\mathrm{DBP}(\mathrm{mmHg})$ & $73.72 \pm 8.86$ & $75.79 \pm 8.94$ & 0.000 & 0.042 \\
\hline $\mathrm{AOV}(\mathrm{ml})$ & $7.65 \pm 2.89$ & $10.11 \pm 4.09$ & 0.000 & 0.000 \\
\hline \multicolumn{5}{|l|}{ Hormonal levels } \\
\hline TT (nmol/L) & $1.51 \pm 0.53$ & $2.35 \pm 0.77$ & 0.000 & 0.000 \\
\hline SHBG (nmol/L) & $54.59 \pm 27.16$ & $35.12 \pm 26.84$ & 0.000 & 0.000 \\
\hline FAl & $3.40 \pm 2.11$ & $9.78 \pm 7.07$ & 0.000 & 0.000 \\
\hline LH/FSH & $1.25 \pm 1.25$ & $2.30 \pm 1.28$ & 0.000 & 0.000 \\
\hline \multicolumn{5}{|l|}{ Metabolic profile } \\
\hline Fasting Ins (pmol/L) & $63.82 \pm 35.26$ & $106.01 \pm 72.79$ & 0.000 & 0.000 \\
\hline Fasting Glu (mmol/L) & $5.24 \pm 0.47$ & $5.36 \pm 0.86$ & 0.002 & 0.900 \\
\hline HOMA-IR & $2.23 \pm 1.30$ & $3.81 \pm 3.09$ & 0.000 & 0.000 \\
\hline $\mathrm{TG}(\mathrm{mmol} / \mathrm{L})$ & $1.04 \pm 0.88$ & $1.44 \pm 1.39$ & 0.000 & 0.000 \\
\hline $\mathrm{TC}(\mathrm{mmol} / \mathrm{L})$ & $4.25 \pm 0.71$ & $4.43 \pm 0.82$ & 0.000 & 0.000 \\
\hline HDL-C (mmol/L) & $1.51 \pm 0.32$ & $1.38 \pm 0.34$ & 0.000 & 0.002 \\
\hline LDL-C (mmol/L) & $2.36 \pm 0.63$ & $2.57 \pm 0.76$ & 0.000 & 0.000 \\
\hline \multicolumn{5}{|l|}{ Oxidative stress parameters } \\
\hline TOS (nmol $\mathrm{H}_{2} \mathrm{O}_{2}$ Equiv./mL) & $11.24 \pm 5.32$ & $14.92 \pm 10.77$ & 0.000 & 0.000 \\
\hline T-AOC (U/ml/min) & $14.50 \pm 2.69$ & $15.80 \pm 3.06$ & 0.000 & 0.000 \\
\hline OSI & $0.80 \pm 0.41$ & $0.99 \pm 0.79$ & 0.000 & 0.000 \\
\hline $\mathrm{MDA}(\mathrm{nmol} / \mathrm{ml})$ & $3.66 \pm 1.07$ & $4.34 \pm 1.31$ & 0.000 & 0.000 \\
\hline
\end{tabular}

Values are presented as mean $\pm S D$.

$A O V$, average ovarian volume; $B M I$, body mass index; $D B P$, diastolic blood pressure; FAl, free androgen index; F-G score, Ferriman-Gallwey score; FSH, follicle-stimulating hormone; Glu, glucose; HDL-C, high-density lipoprotein cholesterol; HOMA index, homeostatic model assessment of insulin resistance; Ins, insulin; LDL-C, low-density lipoprotein cholesterol; LH, luteinizing hormone; MDA, malondialdehyde; OSI, oxidative stress index; SBP, systolic blood pressure; SHBG, sex hormone-binding globulin; T-AOC, total antioxidant capacity; TC, total cholesterol; TG, triglycerides; TOS, total oxidant status; $\Pi$, total testosterone.

$P^{a}$, All comparisons of parameters were corrected for differences in age and BMI between the 2 groups except the parameters of age and BMI.

\section{Associations of GPX1 P198L and CAT C-262T, GPX1 P198L and SOD2 A16V, and CAT C-262T and SOD2 A16V Genotype Combinations With PCOS}

Because the sample sizes of the GPX1 198LL, CAT-262TT, or SOD2 AA homozygotes were too small, we combined them into heterozygous subgroups.

As shown in Table 3, for the GPX1 P198L and CAT C$262 T$ genotype combinations, the frequencies of the $P L+L L / C C$ combined genotype were $12.8 \%$ in patients with PCOS and significantly higher than $7.7 \%$ in the control women. The multinomial logistic regression model including age and BMI as covariates showed that the $P L+L L / C C$ combined genotype
TABLE 2 | Frequencies of GPX P198L (Pro198Leu) and CAT C-262T genotype and allele in PCOS patients compared with control women.

$\begin{array}{cccc}\text { Controls } & \text { PCOS } & x^{2} & P \\ (n=535) & (n=654) & & \end{array}$

\begin{tabular}{cccccc}
\hline Genotype & & & & & \\
GPx 198 & $P P$ & $490(91.6 \%)$ & $562(85.9 \%)$ & & \\
& $P L$ & $43(8.0 \%)$ & $88(13.5 \%)$ & & \\
CAT-262 & L & $2(0.4 \%)$ & $4(0.6 \%)$ & 9.235 & 0.010 \\
& $C C$ & $499(93.3 \%)$ & $614(93.9 \%)$ & & \\
Allele frequency & $C T$ & $35(6.5 \%)$ & $38(5.8 \%)$ & & \\
GPx 198 & $T$ & $1(0.2 \%)$ & $2(0.3 \%)$ & 0.433 & 0.805 \\
& $P$ & 0.956 & 0.927 & & \\
CAT-262 & $L$ & 0.044 & 0.073 & 9.043 & $0.003^{*}$ \\
& $C$ & 0.965 & 0.968 & & \\
& $T$ & 0.035 & 0.032 & 0.112 & 0.738 \\
\hline
\end{tabular}

Data of genotype are presented as number (\%) of patients or controls.

${ }^{*}$ Odds ratio $(O R)=1.671,95 \%$ confidence interval $(C l): 1.190-2.346$.

is a risk factor for PCOS $(\mathrm{OR}=1.745,95 \% \mathrm{CI}$ : $1.128-2.701$, $P=0.012$ ) when the $P P / C C$ combined genotypes as the reference category.

For the GPX1 P198L and SOD2 A16V genotype combinations, the frequencies of the $P L+L L / A V+A A$ combined genotypes were $4.7 \%$ in patients with PCOS, and significantly higher than $1.1 \%$ in the control women $(P=0.001$, Table 3$)$. The multinomial logistic regression model including age and BMI as covariates showed that compared with the subjects carrying the $P P / V V$ genotype, the individuals carrying both the GPx1 $L(P L+L L$ genotype) and SOD2 $A(A V+A A$ genotype) alleles have the highest risk of PCOS (OR $=5.774,95 \% \mathrm{CI}: 2.243-14.863, P<0.001$, Table 3).

For the CAT C-262T and SOD2 A16V genotype combinations, the frequencies of the $C C / A V+A A$ combined genotype were $24.8 \%$ in patients with PCOS and relatively high than $19.8 \%$ in the control women $(P=0.058$, Table 3$)$. The multinomial logistic regression model including age and $\mathrm{BMI}$ as covariates showed that the $C C / A V+A A$ combined genotype is a risk factor for PCOS $(\mathrm{OR}=1.421,95 \% \mathrm{CI}: 1.039-1.942, P=0.028)$ when the $C C / V V$ combined genotypes as the reference category.

\section{Effects of GPx1 P198L and CAT C-262T Genetic Variants on Clinical, Hormonal, Metabolic, and Oxidative Stress Parameters}

We further analyzed effects of GPx1 P198L and CAT C-262T genetic variants on oxidative stress as well as clinical, hormonal, and metabolic parameters in PCOS patients and control women.

As shown in Table 4, compared with the $P P$ genotype subgroup, the $L$ allele carriers ( $P L+L L$ genotype) of the GPx1 $P 198 L$ polymorphism had significantly lower acne grade score $(P=0.004)$ and tended to have increased waist circumference, average ovarian volume, and MDA levels $(P<0.07)$ in the patients with PCOS. The controls with the $L$ allele had higher SBP 
TABLE 3 | Frequencies of combined genotypes of GPx1 P198L and CAT C-262T, GPX1 P198L and SOD2 A16V, and CAT C-262T and SOD2 A16V in patients with $\mathrm{PCOS}$ and the control women.

\begin{tabular}{|c|c|c|c|c|c|}
\hline $\begin{array}{l}\text { Genotype } \\
\text { combinations }\end{array}$ & $\begin{array}{l}\text { Controls } \\
(n=535)\end{array}$ & $\begin{array}{c}\text { PCOS } \\
(n=654)\end{array}$ & OR & $95 \% \mathrm{Cl}$ & $P$ \\
\hline \multicolumn{6}{|c|}{ GPx1 P198L and CAT C-262T* } \\
\hline$P P / C C$ & 458 (85.6\%) & 530 (81.0\%) & 1.00 & - & - \\
\hline$P L+L L / C C$ & 41 (7.7\%) & 84 (12.8\%) & 1.745 & $1.128-2.701$ & 0.012 \\
\hline$P P / C T+T T$ & 32 (6.0\%) & 32 (4.9\%) & 0.828 & $0.468-1.464$ & 0.516 \\
\hline$P L+L L / C T+T T$ & $4(0.7 \%)$ & $(1.2 \%)$ & 1.665 & $0.438-6.331$ & 0.454 \\
\hline \multicolumn{6}{|c|}{ GPx1 P198L and SOD2 A16V** } \\
\hline$P P / W$ & 384 (71.8\%) & 417 (63.8\%) & 1.00 & - & - \\
\hline$P L+L L N$ & 39 (7.3\%) & 61 (9.3\%) & 1.333 & $0.829-2.145$ & 0.236 \\
\hline$P P / A V+A A$ & 106 (19.8\%) & 145 (22.2\%) & 1.300 & $0.943-1.791$ & 0.109 \\
\hline$P L+L L / A V+A A$ & $6(1.1 \%)$ & 31 (4.7\%) & 5.774 & $2.243-14.863$ & 0.000 \\
\hline \multicolumn{6}{|c|}{ CAT C-262T and SOD2 A16V*** } \\
\hline CC/W & 393 (73.5\%) & 452 (69.1\%) & 1.00 & - & - \\
\hline$C C / A V+A A$ & 106 (19.8\%) & $162(24.8 \%)$ & 1.421 & $1.039-1.942$ & 0.028 \\
\hline$C T+T T M W$ & 30 (5.6\%) & $26(4.0 \%)$ & 0.745 & $0.404-1.376$ & 0.347 \\
\hline$C T+T T / A V+A A$ & $6(1.1 \%)$ & $14(2.1 \%)$ & 1.880 & $0.652-5.423$ & 0.243 \\
\hline
\end{tabular}

Data of genotype combinations are presented as number (\%) of patients or controls. ${ }^{*}$ Chi-squared test: $x^{2}=9.558, P=0.023$. Odds ratio $(O R)$ and $95 \%$ confidence interval (Cl) were calculated in a multinomial logistic regression model including age and BMI as covariates, the PP/CC combined genotypes (wild-type) as the reference category.

${ }^{* *}$ Chi-squared test: $x^{2}=17.416, P=0.001$. OR and $95 \% \mathrm{Cl}$ were calculated in a multinomial logistic regression model including age and BMI as covariates, the PPNV combined genotypes (wild-type) as the reference category.

${ }^{\star \star \star}$ Chi-squared test: $x^{2}=7.742, P=0.058 . \mathrm{OR}$ and $95 \% \mathrm{Cl}$ were calculated in a multinomial logistic regression model including age and BMI as covariates, the CCNV combined genotypes (wild-type) as the reference category.

$(P=0.025)$ and tended to have increased OSI $(P=0.084)$ when compared to the controls with the $P P$ genotype.

In addition, compared with the controls with the $C T+T T$ genotype, the controls with the CC genotype of the CAT C-262T polymorphism had higher or relatively high acne grade score and waist circumference $(P<0.08)$ but relatively low F-G score $(P$ $=0.065$, Table 5). No significant differences were observed in clinical, hormonal, and oxidative stress parameters between the $C C$ genotype subgroup and the $C T+T T$ genotype subgroup in the patients with PCOS $(P>0.05$, Table 5).

No significant differences were observed in metabolic indexes between the $P P$ genotype subgroup and the $P L+L L$ genotype subgroup or between the $C C$ genotype subgroup and the $C T+$ $T T$ genotype subgroup in the patients with PCOS or the control women $(P>0.05$, data not shown).

\section{DISCUSSION}

In this study, we show that the GPx1 P198L, but not the CAT $C-262 T$, genetic variant, is associated with a risk of PCOS in Chinese women. We also found that patients carrying the $L$ allele carriers ( $P L+L L$ genotype) of the GPx1 P198L polymorphism had relatively high average ovarian volume, waist circumference, and MDA levels, but lower acne grade score compared with patients carrying the $P P$ genotype, suggesting that increased risk of PCOS in patients with the P198L variant may be potentially linked to ovarian functional abnormalities and increasing levels of oxidative stress in patients. Furthermore, we demonstrated that the individuals carrying both GPX1 198L ( $P L+L L$ genotype) and SOD2 16A (AV+AA genotype) alleles had further increased risk of PCOS compared with the subjects carrying the $P P / V V$ genotype after adjusting age and BMI, implying that the combination of the SOD2 16A and GPX1 198L alleles may have a synergistic effect on the risk of PCOS and patients with PCOS have a genetic susceptibility to oxidative stress.

Oxidative stress plays an important role in the pathogenesis of PCOS $(6,8,36)$. The study of SNPs of antioxidant genes is a helpful method to determine the susceptibility of patients to oxidative stress and elucidate the pathogenesis of disease. CAT and GPx1, located in the peroxisomes and mitochondria, respectively, are primarily intracellular antioxidant enzymes, can detoxify $\mathrm{H}_{2} \mathrm{O}_{2}$ into water, and play a key role in protecting cells against the toxic effects of $\mathrm{H}_{2} \mathrm{O}_{2}(27,40,41)$. Genetic studies have indicated that the $L$ allele of GPX1 P198L polymorphism is associated with decreased enzyme activity of GPxl (13) and is a risk factor for lung cancer among Caucasians (12), breast cancer among Danish women (13), diabetic peripheral neuropathy in the Polish population (11), and coronary artery disease in the Chinese population (42). The $L L$ genotype is also associated with increased risk of bladder cancer in the Turkish population (14) and ulcerative colitis (15). The $C-262 T$ polymorphism in the promoter region of the CAT gene has been reported to affect the expression of CAT gene $(16,18,19)$ and the $T$ allele is associated with lower enzyme activity of CAT although the results reported previously are not entirely consistent $(19,20)$. Several studies have demonstrated that the CC genotype or $C$ allele of $C-262 T$ polymorphism is a risk factor for diabetic neuropathy in type 1 diabetes in the Russian population (43) and male infertility in the Spanish population (22), while the $T$ allele is associated with the risk of increased asthma among Hispanic children (44) and breast cancer (45).

Increased sources of $\mathrm{H}_{2} \mathrm{O}_{2}$ and/or decreased GPxl and/or CAT activities due to genetic variation may result in an increase of $\mathrm{H}_{2} \mathrm{O}_{2}$ both inside and outside cells, relating to the occurrence of some diseases $(27,46,47)$. For example, it has been reported that increasing age, smoking, low serum selenium levels, and the GPX1 P198L and CAT C-262T genetic polymorphisms were significantly associated with prostate disease risk (47). The GPX1 P198L, but not CAT C-262T, genetic polymorphism was associated with non-Hodgkin's lymphoma (48), while the CAT C-262T, but not GPx1 P198L, genetic polymorphism was associated with cerebral palsy after perinatal hypoxic-ischaemic encephalopathy (49). In the present study, we found that the prevalence of the $L$ allele ( $P L+L L$ genotype) was significantly more frequent in patients with PCOS than in the control women. Genotype $(P L+L L)$ remained a significant predictor for PCOS in prognostic models, including age, BMI, HOMAIR, TG, TC, HDL-C, and LDL-C as covariates. Our results suggest that the $L$ allele of GPx1 P198L polymorphism is a genetic risk factor for PCOS in Chinese women. However, our study was unable to prove that the CAT C-262T genetic polymorphism was associated with the risk of PCOS in Chinese women, which was consistent with the report by Salahshoor et al. (28). 
TABLE 4 | Clinical characteristics, hormonal levels, and oxidative stress parameters of the GPX P198L (Pro198Leu) genotypes in PCOS patients and controls.

\begin{tabular}{|c|c|c|c|c|}
\hline & \multicolumn{2}{|c|}{ Controls } & \multicolumn{2}{|c|}{ Pcos } \\
\hline Age (years) & $28.12 \pm 4.17$ & $28.42 \pm 3.97$ & $25.07 \pm 4.15$ & $24.60 \pm 4.58$ \\
\hline $\mathrm{BMI}\left(\mathrm{kg} / \mathrm{m}^{2}\right)$ & $21.14 \pm 2.92$ & $20.88 \pm 2.23$ & $23.28 \pm 4.28$ & $22.94 \pm 4.20$ \\
\hline Waist circumference (cm) & $73.82 \pm 8.37$ & $73.29 \pm 6.90$ & $79.97 \pm 11.45$ & $80.36 \pm 11.32$ \\
\hline Acne grade score & $0.14 \pm 0.34$ & $0.16 \pm 0.37$ & $0.69 \pm 0.92$ & $0.38 \pm 0.77^{b}$ \\
\hline $\mathrm{SBP}(\mathrm{mmHg})$ & $112.61 \pm 11.51$ & $116.50 \pm 10.60^{a}$ & $114.66 \pm 10.81$ & $115.97 \pm 10.09$ \\
\hline $\mathrm{DBP}(\mathrm{mmHg})$ & $73.77 \pm 8.81$ & $73.14 \pm 6.70$ & $75.69 \pm 9.08$ & $76.39 \pm 8.08$ \\
\hline $\mathrm{AOV}(\mathrm{ml})$ & $7.65 \pm 2.99$ & $7.59 \pm 1.71$ & $9.96 \pm 3.89$ & $10.99 \pm 5.03$ \\
\hline \multicolumn{5}{|l|}{ Hormonal levels } \\
\hline \multicolumn{5}{|l|}{ Oxidative stress parameters } \\
\hline TOS (nmol $\mathrm{H}_{2} \mathrm{O}_{2}$ Equiv./mL) & $11.15 \pm 5.18$ & $12.19 \pm 6.61$ & $14.82 \pm 10.72$ & $15.59 \pm 11.12$ \\
\hline T-AOC (U/ml/min) & $14.52 \pm 2.69$ & $14.28 \pm 2.78$ & $15.76 \pm 3.09$ & $15.99 \pm 2.90$ \\
\hline OSI & $0.79 \pm 0.39$ & $0.91 \pm 0.57$ & $0.98 \pm 0.78$ & $1.02 \pm 0.83$ \\
\hline MDA (nmol/ml) & $3.64 \pm 1.06$ & $3.88 \pm 1.22$ & $4.31 \pm 1.23$ & $4.57 \pm 1.71$ \\
\hline
\end{tabular}

Values are presented as mean $\pm S D$.

AOV, average ovarian volume; BMI, body mass index; DBP, diastolic blood pressure; FAl, free androgen index; F-G score, Ferriman-Gallwey score; FSH, follicle-stimulating hormone; LH, luteinizing hormone; MDA, malondialdehyde; OSI, oxidative stress index; SBP, systolic blood pressure; SHBG, sex hormone-binding globulin; T-AOC, total antioxidant capacity; TOS, total oxidant status; TT, total testosterone.

Comparisons of all parameters were corrected for differences in age and BMI between the 2 subgroups except the parameters of age and BMI.

${ }^{a} P<0.05$, compared with the PP genotype subgroup in controls.

${ }^{b} P<0.01$, compared with the PP genotype subgroup in PCOS patients.

It has been shown that 3 genes are active on the pathway of detoxification of reactive oxygen species (ROS) from $\mathrm{O}_{2}^{-}$to $\mathrm{H}_{2} \mathrm{O}_{2}$ (SOD2), and further to $\mathrm{H}_{2} \mathrm{O}$ (GPX1 and CAT), and the genetic variations of these genes may influence the efficiency of this detoxification $(11,16,27)$. Ravn-Haren et al. (13) reported that there is a significant effect of the GPX1 gene P198L variant on enzyme activity and the catalytic activity was lowered $5 \%$ for each additional copy of the variant $L$ allele. Recently, our study found that the $A$ allele SOD2 A16V polymorphism is a genetic risk factor of PCOS in Chinese women (23). The SOD2V $\rightarrow$ $A$ variant has been reported to enhance SOD2 precursor mitochondrial transport and thus increases enzyme activity in the mitochondrial matrix (50), increasing $\mathrm{O}_{2}{ }_{2}^{-}$dismutation and decreasing ROS-mediated toxicity in the mitochondria because membrane permeability $\mathrm{H}_{2} \mathrm{O}_{2}$ easily reaches the cytosol (46). However, increased SOD2 activity may lead to cell damage by $\mathrm{H}_{2} \mathrm{O}_{2}$ overproduction, especially in individuals with a decreased capacity to remove this highly toxic ROS by GPx or CAT $(46,51)$. Based on the association of the GPx1 $198 P \rightarrow L$ variant with decreased enzyme activity (13) and the increased risk of bladder and breast cancer due to the combination of the SOD2 Ala/Ala and GPX1 Leu/Leu genotypes (14, 51), we further analyzed association of SOD2 A16V and GPX1 P198L genotype combinations with the risk of PCOS. The results showed that the individuals carrying the $P L+L L / A V+A A$ combined genotype had further increased risk of PCOS. In addition, we showed that the $P L+L L / A V+A A$ genotype subgroup had higher SBP $(118.96 \pm$ 9.22 vs. $114.57 \pm 10.25$ or $114.78 \pm 11.07 \mathrm{mmHg}, P<0.05)$ and DBP $(79.29 \pm 7.22$ vs. $75.03 \pm 8.15$ or $75.64 \pm 9.84 \mathrm{mmHg}, P$ $<0.05)$, higher or relatively high OSI $(1.25 \pm 1.14$ vs. $0.90 \pm$ 0.59 or $0.87 \pm 0.54, P<0.08$ ), but lower or relatively low T-AOC levels $(14.73 \pm 2.64$ vs. $16.58 \pm 2.85$ or $15.77 \pm 3.34 \mathrm{U} / \mathrm{ml} / \mathrm{min}, P$ $<0.10)$ compared with the $P L+L L / V V$ or $P P / A V+A A$ genotype subgroups in patients with PCOS. Our results suggest that the combination of the SOD2 A and GPX1 L alleles may have a synergistic effect on the risk of PCOS.

We should point out that this study has some limitations. Firstly, given the low frequency of homozygosity of minor alleles, GPX1 198LL, and CAT-262TT, we could not analyze them in the form of subgroups. A larger sample size of patients and controls are needed to properly evaluate dose-dependent genotype characteristics. Secondly, we could not determine GPx and CAT activities. Further study to detect these enzyme activities in the patients with different genotypes may help provide clues to the mechanisms responsible for the genetic association.

In summary, we have demonstrated that the GPx1 P198L, but not $C A T C-262 T$ genetic polymorphism is associated with the risk of PCOS in Chinese women. Our findings also suggest that the P198L variant increasing the risk of PCOS may be implicated in ovarian functional abnormalities and increased oxidative stress in 
TABLE 5 | Clinical characteristics, hormonal levels, and oxidative stress parameters of the CAT C-262T genotypes in PCOS patients and controls.

\begin{tabular}{|c|c|c|c|c|}
\hline & \multicolumn{2}{|c|}{ Controls } & \multicolumn{2}{|c|}{ PCOS } \\
\hline & $\begin{array}{c}C C \\
(n=499)\end{array}$ & $\begin{array}{c}C T+T T \\
(n=35+1)\end{array}$ & $\begin{array}{c}C C \\
(n=614)\end{array}$ & $\begin{array}{c}C T+T T \\
(n=38+2)\end{array}$ \\
\hline Age (years) & $28.20 \pm 4.07$ & $27.44 \pm 5.15$ & $25.05 \pm 4.24$ & $24.33 \pm 3.70$ \\
\hline $\mathrm{BMI}\left(\mathrm{kg} / \mathrm{m}^{2}\right)$ & $21.14 \pm 2.88$ & $20.84 \pm 2.59$ & $23.25 \pm 4.26$ & $22.97 \pm 4.40$ \\
\hline Waist circumference (cm) & $73.82 \pm 8.37$ & $71.46 \pm 8.34$ & $80.10 \pm 11.44$ & $78.41 \pm 11.28$ \\
\hline Waist-to-hip ratio & $0.82 \pm 0.06$ & $0.80 \pm 0.06$ & $0.85 \pm 0.07$ & $0.84 \pm 0.07$ \\
\hline F-G score & $0.24 \pm 0.67$ & $0.47 \pm 1.32$ & $1.73 \pm 2.06$ & $1.93 \pm 2.14$ \\
\hline Acne grade score & $0.15 \pm 0.37$ & $0.00 \pm 0.00^{a}$ & $0.64 \pm 0.90$ & $0.83 \pm 0.96$ \\
\hline $\mathrm{SBP}(\mathrm{mmHg})$ & $113.07 \pm 11.48$ & $110.79 \pm 11.50$ & $114.82 \pm 10.72$ & $115.26 \pm 10.69$ \\
\hline $\mathrm{DBP}(\mathrm{mmHg})$ & $73.73 \pm 8.71$ & $73.55 \pm 8.00$ & $75.68 \pm 8.83$ & $77.44 \pm 10.41$ \\
\hline $\mathrm{AOV}(\mathrm{ml})$ & $7.58 \pm 2.72$ & $8.27 \pm 4.39$ & $10.05 \pm 3.96$ & $11.13 \pm 5.82$ \\
\hline \multicolumn{5}{|l|}{ Hormonal levels } \\
\hline TT (nmol/L) & $1.50 \pm 0.53$ & $1.66 \pm 0.53$ & $2.35 \pm 0.78$ & $2.36 \pm 0.74$ \\
\hline SHBG (nmol/L) & $54.61 \pm 27.57$ & $54.37 \pm 20.03$ & $34.81 \pm 27.07$ & $40.07 \pm 22.67$ \\
\hline FAl & $3.42 \pm 2.15$ & $3.14 \pm 1.51$ & $9.89 \pm 7.08$ & $7.99 \pm 6.76$ \\
\hline LH/FSH & $1.27 \pm 1.27$ & $1.10 \pm 0.81$ & $2.31 \pm 1.30$ & $2.11 \pm 0.91$ \\
\hline \multicolumn{5}{|l|}{ Oxidative stress parameters } \\
\hline TOS (nmol $\mathrm{H}_{2} \mathrm{O}_{2}$ Equiv./mL) & $11.17 \pm 5.30$ & $12.14 \pm 5.49$ & $14.85 \pm 10.65$ & $16.16 \pm 12.56$ \\
\hline T-AOC (U/ml/min) & $14.54 \pm 2.75$ & $13.97 \pm 1.77$ & $15.77 \pm 3.09$ & $16.13 \pm 2.62$ \\
\hline OSI & $0.80 \pm 0.41$ & $0.88 \pm 0.37$ & $0.99 \pm 0.80$ & $1.00 \pm 0.69$ \\
\hline MDA (nmol/ml) & $3.66 \pm 1.08$ & $3.64 \pm 1.02$ & $4.35 \pm 1.28$ & $4.21 \pm 1.76$ \\
\hline
\end{tabular}

Values are presented as the mean $\pm S D$.

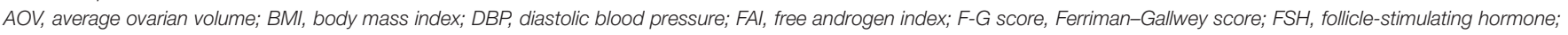

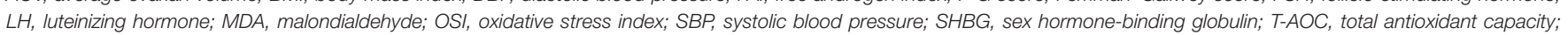
TOS, total oxidant status; $T$, total testosterone.

Comparisons of all parameters were corrected for differences in age and BMI between the 2 subgroups except the parameters of age and BMI.

${ }^{a} P<0.05$, compared with the CC genotype subgroup in controls.

patients. Furthermore, we indicate that, compared with women carrying both PP genotype of GPx1 P198L and VV genotype of SOD2 A16V polymorphisms, the women carrying both the $198 \mathrm{~L}$ allele and $16 \mathrm{~A}$ allele had higher risk of PCOS. Our studies suggest that antioxidant enzyme gene variation may increase the sensitivity of patients to oxidative stress and thus contribute to the pathogenesis of PCOS.

\section{DATA AVAILABILITY STATEMENT}

The datasets for this study can be found at the found here: CAT C-262T (rs1001179): https:/www.ncbi.nlm.nih.gov/snp/ rs1001179, GPx1 P198L (rs1050450): https://www.ncbi.nlm.nih. gov/snp/rs1050450, SOD2 A16V (rs4880): https://www.ncbi. nlm.nih.gov/snp/rs4880.

\section{ETHICS STATEMENT}

The study was approved by the Institutional Review Board of the West China Second University Hospital, Sichuan University (2014-014 to PF). All of the participants provided their written informed consent in accordance with the Helsinki Declaration of ethical conduct in research.

\section{AUTHOR CONTRIBUTIONS}

PF conceived and designed the experiments, analyzed the data, and revised the paper. YS performed experiments and wrote the paper. HL and WH were responsible for patient screening. YG and YS collected samples and clinical data. QL and LG helped with the experiments. SL and HB helped with the experiments and revised the paper. All authors read and approved the final manuscript.

\section{FUNDING}

This work was funded by the Chinese National Natural Science Foundation (81370681) and the Program for Changjiang Scholars and Innovative Research Team in University, Ministry of Education (IRT0935).

\section{ACKNOWLEDGMENTS}

We thank women with or without PCOS who donated blood samples for this study. We are thankful to You Li, De Hua Wan, Qi Song, Feng Zhang, Ying Wang, Jinxia Zhang, Yujin Zhang, and Renjiao Zhang for their help with the sample collection. 


\section{REFERENCES}

1. Skiba MA, Islam RM, Bell RJ, Davis SR. Understanding variation in prevalence estimates of polycystic ovary syndrome: a systematic review and meta-analysis. Hum Reprod Update. (2018) 24:694-709. doi: 10.1093/humupd/dmy022

2. Brakta S, Lizneva D, Mykhalchenko K, Imam A, Walker W, Diamond MP, et al. Perspectives on polycystic ovary syndrome: is polycystic ovary syndrome research underfunded? J Clin Endocrinol Metab. (2017) 102:4421-7. doi: 10.1210/jc.2017-01415

3. Rosenfield RL, Ehrmann DA. The pathogenesis of polycystic ovary syndrome (PCOS): the hypothesis of PCOS as functional ovarian hyperandrogenism revisited. Endocr Rev. (2016) 37:467-520. doi: 10.1210/er.2015-1104

4. Zhang J, Fan P, Liu H, Bai H, Wang Y, Zhang F. Apolipoprotein A-I and B levels, dyslipidemia and metabolic syndrome in south-west Chinese women with PCOS. Hum Reprod. (2012) 27:2484-93. doi: 10.1093/humrep/des191

5. Lim SS, Davies MJ, Norman RJ, Moran LJ. Overweight, obesity and central obesity in women with polycystic ovary syndrome: a systematic review and meta-analysis. Hum Reprod Update. (2012) 18:618-37. doi: 10.1093/humupd/dms030

6. Zhang R, Liu H, Bai H, Zhang Y, Liu Q, Guan L, et al. Oxidative stress status in Chinese women with different clinical phenotypes of polycystic ovary syndrome. Clin Endocrinol. (2017) 86:88-96. doi: 10.1111/cen.13171

7. Zhao Y, Zhang C, Huang Y, Yu Y, Li R, Li M, et al. Up-regulated expression of WNT5a increases inflammation and oxidative stress via PI3K/AKT/NFkappaB signaling in the granulosa cells of PCOS patients. J Clin Endocrinol Metab. (2015) 100:201-11. doi: 10.1210/jc.2014-2419

8. Murri M, Luque-Ramirez M, Insenser M, Ojeda-Ojeda M, Escobar-Morreale HF. Circulating markers of oxidative stress and polycystic ovary syndrome (PCOS): a systematic review and meta-analysis. Hum Reprod Update. (2013) 19:268-88. doi: 10.1093/humupd/dms059

9. Kakoly NS, Khomami MB, Joham AE, Cooray SD, Misso ML, Norman RJ, et al. Ethnicity, obesity and the prevalence of impaired glucose tolerance and type 2 diabetes in PCOS: a systematic review and meta-regression. Hum Reprod Update. (2018) 24:455-67. doi: 10.1093/humupd/dmy007

10. de Groot PC, Dekkers OM, Romijn JA, Dieben SW, Helmerhorst FM. PCOS, coronary heart disease, stroke and the influence of obesity: a systematic review and meta-analysis. Hum Reprod Update. (2011) 17:495-500. doi: 10.1093/humupd/dmr001

11. Buraczynska M, Buraczynska K, Dragan M, Ksiazek A. Pro198Leu polymorphism in the glutathione peroxidase 1 gene contributes to diabetic peripheral neuropathy in type 2 diabetes patients. Neuromolecular Med. (2017) 19:147-53. doi: 10.1007/s12017-016-8438-2

12. Ratnasinghe D, Tangrea JA, Andersen MR, Barrett MJ, Virtamo J, Taylor PR, et al. Glutathione peroxidase codon 198 polymorphism variant increases lung cancer risk. Cancer Res. (2000) 60:6381-3.

13. Ravn-Haren G, Olsen A, Tjonneland A, Dragsted LO, Nexo BA, Wallin H, et al. Associations between GPX1 Pro198Leu polymorphism, erythrocyte GPX activity, alcohol consumption and breast cancer risk in a prospective cohort study. Carcinogenesis. (2006) 27:820-5. doi: 10.1093/carcin/bgi267

14. Kucukgergin C, Sanli O, Amasyali AS, Tefik T, Seckin S. Genetic variants of MnSOD and GPX1 and susceptibility to bladder cancer in a Turkish population. Med Oncol. (2012) 29:1928-34. doi: 10.1007/s12032-011-0057-z

15. Costa Pereira C, Duraes C, Coelho R, Gracio D, Silva M, Peixoto A, et al. Association between polymorphisms in antioxidant genes and inflammatory bowel disease. PLoS ONE. (2017) 12:e0169102. doi: 10.1371/journal.pone.0169102

16. Wenten M, Gauderman WJ, Berhane K, Lin PC, Peters J, Gilliland FD. Functional variants in the catalase and myeloperoxidase genes, ambient air pollution, and respiratory-related school absences: an example of epistasis in gene-environment interactions. Am J Epidemiol. (2009) 170:1494-501. doi: 10.1093/aje/kwp310

17. Goth L, Eaton JW. Hereditary catalase deficiencies and increased risk of diabetes. Lancet. (2000) 356:1820-1. doi: 10.1016/s0140-6736(00)03238-4

18. Forsberg L, Lyrenas L, de Faire U, Morgenstern R. A common functional C$\mathrm{T}$ substitution polymorphism in the promoter region of the human catalase gene influences transcription factor binding, reporter gene transcription and is correlated to blood catalase levels. Free Radic Biol Med. (2001) 30:500-5. doi: 10.1016/s0891-5849(00)00487-1

19. Goulas A, Agapakis D, Apostolidis A, Gouda D, Anastassiadis S, Trakatelli C, et al. Association of the common catalase gene polymorphism rs1001179 with glycated hemoglobin and plasma lipids in hyperlipidemic patients. Biochem Genet. (2017) 55:77-86. doi: 10.1007/s10528-016-9777-2

20. Goth L, Nagy T, Kosa Z, Fejes Z, Bhattoa HP, Paragh G, et al. Effects of rs769217 and rs1001179 polymorphisms of catalase gene on blood catalase, carbohydrate and lipid biomarkers in diabetes mellitus. Free Radic Res. (2012) 46:1249-57. doi: 10.3109/10715762.2012.702899

21. Hu J, Feng F, Zhu S, Sun L, Li G, Jiang N, et al. Catalase C-262T polymorphism and risk of prostate cancer: evidence from meta-analysis. Gene. (2015) 558:265-70. doi: 10.1016/j.gene.2015.01.005

22. Garcia Rodriguez A, de la Casa M, Johnston S, Gosalvez J, Roy R. Association of polymorphisms in genes coding for antioxidant enzymes and human male infertility. Ann Hum Genet. (2019) 83:63-72. doi: 10.1111/ahg.12286

23. Liu Q, Liu H, Bai H, Huang W, Zhang R, Tan J, et al. Association of SOD2 A16V and PON2 S311C polymorphisms with polycystic ovary syndrome in Chinese women. J Endocrinol Invest. (2019) 42:909-21. doi: 10.1007/s40618-018-0999-5

24. Wang Y, Liu H, Fan P, Bai H, Zhang J, Zhang F. Evidence for association between paraoxonase 1 gene polymorphisms and polycystic ovarian syndrome in southwest Chinese women. Eur J Endocrinol. (2012) 166:877-85. doi: 10.1530/eje-11-0986

25. Fan P, Liu HW, Wang XS, Zhang F, Song Q, Li Q, et al. Identification of the G994T polymorphism in exon 9 of plasma platelet-activating factor acetylhydrolase gene as a risk factor for polycystic ovary syndrome. Hum Reprod. (2010) 25:1288-94. doi: 10.1093/humrep/deq047

26. Banescu C, Trifa AP, Voidazan S, Moldovan VG, Macarie I, Benedek Lazar E, et al. CAT, GPX1, MnSOD, GSTM1, GSTT1, and GSTP1 genetic polymorphisms in chronic myeloid leukemia: a case-control study. Oxid Med Cell Longev. (2014) 2014:875861. doi: 10.1155/2014/875861

27. Fukai T, Ushio-Fukai M. Superoxide dismutases: role in redox signaling, vascular function, and diseases. Antioxid Redox Signal. (2011) 15:1583-606. doi: 10.1089/ars.2011.3999

28. Salahshoor MR, Sohrabi M, Jalili F, Jalili P, Rezavand N, Haghnazari L, et al. No evidence for a major effect of three common polymorphisms of the GPx1, MnSOD, and CAT genes on PCOS susceptibility. J Cell Biochem. (2019) 120:2362-9. doi: 10.1002/jcb.27564

29. The Rotterdam ESHRE/ASRM-Sponsored PCOS Consensus Workshop Group. Revised 2003 consensus on diagnostic criteria and long-term health risks related to polycystic ovary syndrome. Fertil Steril. (2004) 81:19-25. doi: 10.1016/j.fertnstert.2003.10.004

30. Teede HJ, Misso ML, Costello MF, Dokras A, Laven J, Moran L, et al. Recommendations from the international evidence-based guideline for the assessment and management of polycystic ovary syndrome. Hum Reprod. (2018) 33:1602-18. doi: 10.1093/humrep/dey256

31. Zhang Y, Liu H, He J, Xu K, Bai H, Wang Y, et al. Lactonase activity and status of paraoxonase 1 in Chinese women with polycystic ovarian syndrome. Eur J Endocrinol. (2015) 172:391-402. doi: 10.1530/EJE-14-0863

32. The Amsterdam ESHRE/ASRM-Sponsored 3rd PCOS Consensus Workshop Group. Consensus on women's health aspects of polycystic ovary syndrome (PCOS). Hum Reprod. (2012) 27:14-24. doi: 10.1093/humrep/der396

33. Yuan XH, Fan YY, Yang CR, Gao XR, Zhang LL, Hu Y, et al. Progesterone amplifies oxidative stress signal and promotes $\mathrm{NO}$ production via $\mathrm{H} 2 \mathrm{O} 2$ in mouse kidney arterial endothelial cells. J Steroid Biochem Mol Biol. (2016) 155 (Pt A):104-11. doi: 10.1016/j.jsbmb.2015.09.029

34. Wassmann K, Wassmann S, Nickenig G. Progesterone antagonizes the vasoprotective effect of estrogen on antioxidant enzyme expression and function. Circ Res. (2005) 97:1046-54. doi: 10.1161/01.RES.0000188212.57180.55

35. Zhang R, Liu Q, Liu H, Bai H, Zhang Y, Guan L, et al. Effects of apoC1 genotypes on the hormonal levels, metabolic profile and PAF-AH activity in Chinese women with polycystic ovary syndrome. Lipids Health Dis. (2018) 17:77. doi: 10.1186/s12944-018-0725-5

36. Zhang J, Zhang Y, Liu H, Bai H, Wang Y, Jiang C, et al. Antioxidant properties of high-density lipoproteins are impaired in women 
with polycystic ovary syndrome. Fertil Steril. (2015) 103:1346-54. doi: 10.1016/j.fertnstert.2015.02.024

37. Robert Y, Dubrulle F, Gaillandre L, Ardaens Y, Thomas-Desrousseaux P, Lemaitre L, et al. Ultrasound assessment of ovarian stroma hypertrophy in hyperandrogenism and ovulation disorders: visual analysis versus computerized quantification. Fertil Steril. (1995) 64:307-12.

38. Kasznicki J, Sliwinska A, Kosmalski M, Merecz A, Majsterek I, Drzewoski J. Genetic polymorphisms. (Pro197Leu of Gpx1, +35A/C of SOD1,-262C/T of CAT), the level of antioxidant proteins (GPx1, SOD1, CAT) and the risk of distal symmetric polyneuropathy in Polish patients with type 2 diabetes mellitus. Adv Med Sci. (2016) 61:123-9. doi: 10.1016/j.advms.2015.10.006

39. Jamhiri I, Saadat I, Omidvari S. Genetic polymorphisms of superoxide dismutase-1 A251G and catalase C-262T with the risk of colorectal cancer. Mol Biol Res Commun. (2017) 6:85-90. doi: 10.22099/mbrc.2017.4059

40. Sousa VC, Carmo RF, Vasconcelos LR, Aroucha DC, Pereira LM, Moura P, et al. Association of catalase and glutathione peroxidase 1 polymorphisms with chronic hepatitis C outcome. Ann Hum Genet. (2016) 80:145-53. doi: 10.1111/ahg.12152

41. Cyr AR, Hitchler MJ, Domann FE. Regulation of SOD2 in cancer by histone modifications and $\mathrm{CPG}$ methylation: closing the loop between redox biology and epigenetics. Antioxid Redox Signal. (2013) 18:1946-55. doi: 10.1089 /ars.2012.4850

42. Tang NP, Wang LS, Yang L, Gu HJ, Sun QM, Cong RH, et al. Genetic variant in glutathione peroxidase 1 gene is associated with an increased risk of coronary artery disease in a Chinese population. Clin Chim Acta. (2008) 395:89-93. doi: 10.1016/j.cca.2008.05.013

43. Chistiakov DA, Zotova EV, Savost'anov KV, Bursa TR, Galeev IV, Strokov IA, et al. The $262 \mathrm{~T}>\mathrm{C}$ promoter polymorphism of the catalase gene is associated with diabetic neuropathy in type 1 diabetic Russian patients. Diabetes Metab. (2006) 32:63-8. doi: 10.1016/s1262-3636(07)70248-3

44. Islam T, McConnell R, Gauderman WJ, Avol E, Peters JM, Gilliland FD. Ozone, oxidant defense genes, and risk of asthma during adolescence. Am J Respir Crit Care Med. (2008) 177:388-95. doi: 10.1164/rccm.200706-863OC

45. Ahn J, Gammon MD, Santella RM, Gaudet MM, Britton JA, Teitelbaum SL, et al. Associations between breast cancer risk and the catalase genotype, fruit and vegetable consumption, and supplement use. Am J Epidemiol. (2005) 162:943-52. doi: 10.1093/aje/kwi306
46. Bresciani G, Cruz IB, de Paz JA, Cuevas MJ, Gonzalez-Gallego J. The MnSOD Ala16Val SNP: relevance to human diseases and interaction with environmental factors. Free Radic Res. (2013) 47:781-92. doi: 10.3109/10715762.2013.836275

47. Karunasinghe N, Han DY, Goudie M, Zhu S, Bishop K, Wang A, et al. Prostate disease risk factors among a New Zealand cohort. J Nutrigenet Nutrigenomics. (2012) 5:339-51. doi: 10.1159/000 346279

48. Lightfoot TJ, Skibola CF, Smith AG, Forrest MS, Adamson PJ, Morgan GJ, et al. Polymorphisms in the oxidative stress genes, superoxide dismutase, glutathione peroxidase and catalase and risk of non-Hodgkin's lymphoma. Haematologica. (2006) 91: $1222-7$.

49. Esih K, Goricar K, Dolzan V, Rener-Primec Z. The association between antioxidant enzyme polymorphisms and cerebral palsy after perinatal hypoxic-ischaemic encephalopathy. Eur J Paediatr Neurol. (2016) 20:704-8. doi: 10.1016/j.ejpn.2016.05.018

50. Sutton A, Khoury H, Prip-Buus C, Cepanec C, Pessayre D, Degoul F. The Ala16Val genetic dimorphism modulates the import of human manganese superoxide dismutase into rat liver mitochondria. Pharmacogenetics. (2003) 13:145-57. doi: 10.1097/01.fpc.0000054067.64 $000.8 \mathrm{f}$

51. Cox DG, Tamimi RM, Hunter DJ. Gene x Gene interaction between MnSOD and GPX-1 and breast cancer risk: a nested case-control study. BMC Cancer. (2006) 6:217. doi: 10.1186/1471-2407-6-217

Conflict of Interest: The authors declare that the research was conducted in the absence of any commercial or financial relationships that could be construed as a potential conflict of interest.

Copyright $\odot 2019$ Sun, Li, Liu, Gong, Bai, Huang, Liu, Guan and Fan. This is an open-access article distributed under the terms of the Creative Commons Attribution License (CC BY). The use, distribution or reproduction in other forums is permitted, provided the original author(s) and the copyright owner(s) are credited and that the original publication in this journal is cited, in accordance with accepted academic practice. No use, distribution or reproduction is permitted which does not comply with these terms. 